\title{
Mobility of Nanoparticles Generated by Femtosecond Laser Ablation in Liquids and Its Application to Surface Patterning
}

\author{
Ana MENÉNDEZ-MANJÓN ${ }^{1}$, Jurij JAKOBI ${ }^{1}$, Kerstin SCHWABE ${ }^{2}$, Joachim K. KRAUSS ${ }^{2}$, \\ Stephan BARCIKOWSKI ${ }^{1}$ \\ ${ }^{1}$ Laser Zentrum Hannover e.V., Hollerithallee 8, D- 30419 Hannover, Germany \\ E-mail: s.barcikowski@lzh.de \\ ${ }^{2}$ Medizinische Hochschule Hannover, Carl-Neuberg-Str. 1, D 30625 Hannover, Germany
}

\begin{abstract}
Metal nanoparticles have been generated in deionized water and acetone by femtosecond-pulsed laser ablation. The expansion velocity was measured in-process in dependence on pulse energy using particle tracking image velocimetry. Particle size distributions of silver were determined at periodic time intervals during the generation process, reaching stabilization level around $50 \mathrm{~nm}$ after $5 \cdot 10^{5}$ pulses. Electrophoretic mobility of laser-generated metal nanoparticles was studied by laser scattering velocimetry and the electrophoretic deposition of charged nanoparticles was used to generate nanoparticle-derived nanostructures on 3-dimensional microstructured surfaces.
\end{abstract}

DOI: $10.2961 / \mathrm{jlmn} .2009 .02 .0004$

Keywords: Laser ablation, nanoparticles, electrodeposition, nanostructuring

The irradiation of solid targets with laser pulses of fluences above the ablation threshold leads to material removal forming nanoparticles. Depending on the irradiation conditions different ablation mechanism take place. Molecular dynamics simulation has shown in different fluence regimes two well differentiated phenomena like phase explosion or phase separation [1]. In this way the resulting particle size distribution can be controlled by laser parameters such as fluence or wavelength $[2,3]$. However, delivering pulses through a liquid onto a target in a controlled manner is challenging. Interaction of the laser with the medium leads to disturbing effects on the ablation process such as optical breakdown [4] and cavitation bubble formation. Moreover, ultrashort laser pulses easily reach intensities needed to induce non-linear effects in the liquid, leading to self-focusing and filamentation [5].

For a better comprehension of influence of the focusing conditions on the ablation, a silver target placed under a deionized water column of $6.5 \mathrm{~mm}$ was ablated by a $800 \mathrm{~nm}$ wavelength, $120 \mathrm{fs}$ pulsed laser, working at a repetition rate of $5 \mathrm{kHz}$ and $2.09 \mathrm{~W}$. A lens of $100 \mathrm{~mm}$ focal length was used to focus the laser on the target. Simple microscopic studies of 20 ablated lines (scan direction from left to right) show the effect of bubbles on the process. Figure 1 shows the ablated patterns under different focus positions related to the beam waist in air. As origin of $\mathrm{z}$ axis (target positioning), we took the plane at which the laser beam is focused on the target surface. Placing the target toward the lens is noted with negative $\mathrm{z}$ values. Positive $\mathrm{z}$ values denote the situation of the target behind the focal plane.

Formation of cavitation bubbles around the ablation plume decreases the productivity due to scattering of the incident laser beam. This can be seen in figure $1 \mathrm{~b}$, at the border of the ablated pattern, where bubbles stuck to the surface leave a circular non-irradiated mark. By defocusing the laser beam, the optical breakdown of water and bubble formation can be avoided. Placing the focus under the target (Figure 1a), the pulse energy is not bundled and the ablation is not efficient. If the focus is placed above the target, the propagation length of the beam can be large enough to make the beam filamentate [6] (Figure 1c).

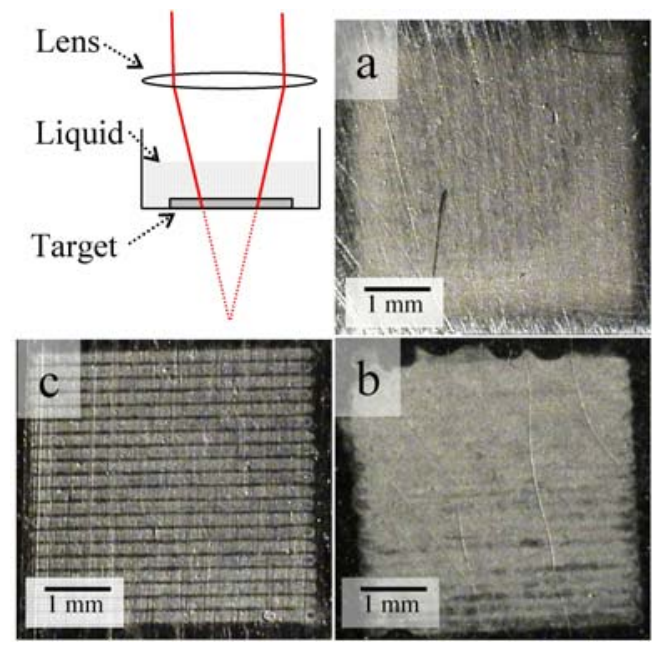

Fig 1 Lines ablated on silver under a $6.5 \mathrm{~mm}$ water column. Incident fluence and focus positions ( $\mathrm{z}$ ) are $19 \mathrm{~mJ} / \mathrm{cm}^{2}, \mathrm{z}=-18 \mathrm{~mm}$ (a); $269 \mathrm{~mJ} / \mathrm{cm}^{2}, \mathrm{z}=-4.75 \mathrm{~mm}$ (b); and $75 \mathrm{~mJ} / \mathrm{cm}^{2}, \mathrm{z}=+9 \mathrm{~mm}$ (c).

Alternatively, inducing a flow in the liquid achieves that the bubbles formed in the volume and on the target are efficiently removed [7]. The delivery of laser pulses onto the target is more effective which can be observed in the mass loss compared for both configurations. The ablation rate in liquid flow results to be higher by a factor of 15 $(10.6 \mathrm{mg} / \mathrm{h})$ compared to stationary liquid $(0.68 \mathrm{mg} / \mathrm{h})$.

The generation of nanoparticles by laser ablation is a two-step process. At first, a solid target is irradiated by laser pulses which ablate material such as atoms, ions and, under certain conditions, particles. The anisotropic diffu- 
sion of the abated spieces ejected from the plume leads to orthokinetik aggregation. As they are ejected normal to the target, nucleate to form bigger clusters dispersed in the liquid matrix. In gas ambient, pressure can be modified to produce greater expansion of the ablated species and thus controlling the final particle size. Since in liquids, transport phenomena play a significant role in the particle expansion, affecting the nucleation rate of primary clusters and the resulting particle size distribution, it is worth investigating the ejection and diffusion behaviour of the particles after the ablation.

Studies on particle velocimetry have been carried out with a commercial device (LM10, Nanosight Ltd.), modified by us for in-situ laser ablation. This device is originally equipped with a $640 \mathrm{~nm}$ wavelength and $30 \mathrm{~mW}$ laser source, which propagates horizontally within a suspension contained in a $0.97 \mathrm{ml}$ cavity (Figure 2). An integrated window at the top permits the visualization of illuminated particles moving randomly under Brownian motion. A scientific camera, mounted on an optical microscope, is used to record the light scattered by the nanoparticles at a frequency of 30 frames per second. One-to-one particle tracking, in an image field of $106 \times 80 \mu \mathrm{m}$, allows determining the hydrodynamic diameter of a sphere based on its movement rate through Stokes-Einstein equation. In order to study the particle diffusion velocities during the ablation process, a quartz window was added at a side of the chamber, so another laser can be coupled in and can ablate a target placed inside (Figure 2a).

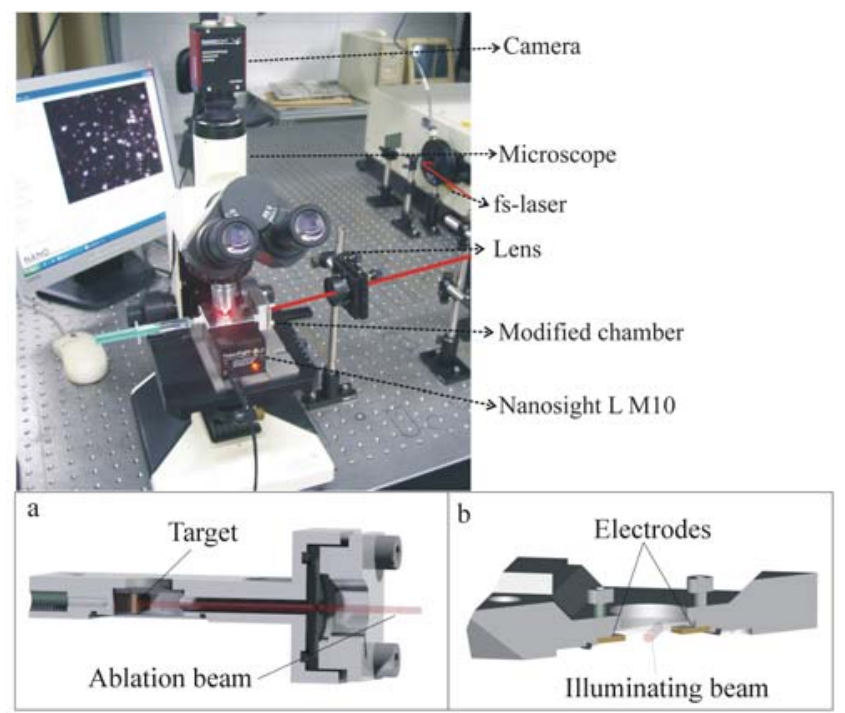

Fig 2 Experimental set-up of modified Nanosight device for ablation experiments. Longitudinal cut of particle tracking and ablation chamber (a) and electrophoretic particle tracking chamber (b).

A Ti:Sapphire laser of 120 fs pulse duration, working at $5 \mathrm{kHz}$, was focused by a $250 \mathrm{~mm}$ lens to ablate the silver target in the liquid reservoir. By changing the pulse energy and fixing the laser spot radius on the target to be $500 \mu \mathrm{m}$, the fluence applied was varied from 5 to $41 \mathrm{~mJ} / \mathrm{cm}^{2}$. Particle velocity distributions have been measured for increasing fluence. The relative maximum of the particle velocity distribution (ejection speed at a distance of $4 \mathrm{~mm}$ from the target) increases almost linearly with the pulse energy (Figure 3a). Velocity distributions of the ejected particles generated at 20 and $160 \mu \mathrm{J}$ pulses are compared in Figure $3 \mathrm{~b}$. The mean velocity increases by a factor $450 \%$. At low incident pulse energies, the velocity distributions are narrow. An increase in the laser pulse energy causes higher production rates, and thus more concentrated colloids. The nanoparticles ejected by the plume interact with the previously generated particles, showing broader velocity distributions at high energy pulses.

During the experiments, it was observed that the nanoparticles ejected from the laser plasma plume turned back to the target. This can be explained as follows: at a primary stage, ablated species are ejected almost normal to the target and are cooled inside the liquid while they propagate from the plasma plume. Stokes frictional resistance and collisions with the ambient molecules and other ablated clusters reduce their velocity until they are suspended in the medium. Because of temperature gradients in the volume, due to plasma plume on the silver plate, convection flow was observed. Particles drift within the liquid, which moves under buoyancy forces, forming a mushroomlike distribution. The same behaviour was observed by Grigoropoulos et al. [8] while ablating films of gold nanoparticles in air. As expected, plume expansion of laser ablated material in vacuum do not shows such a pattern $[9,10]$, as no free convection takes place. In our experimental set-up, as well as by Grigoropoulos et al. experiments, ablation occurred horizontally, so the contribution of gravity has been discarded.
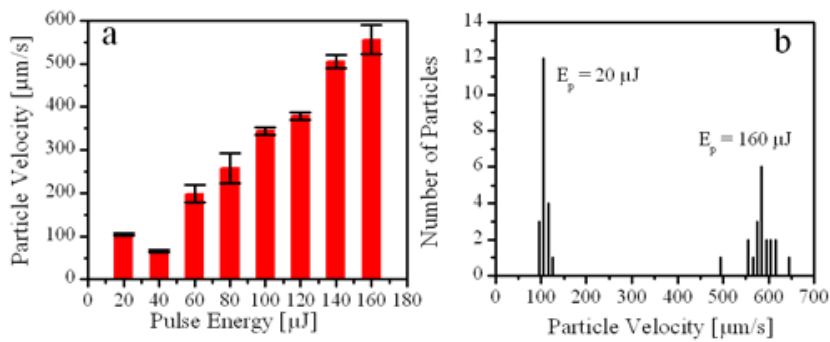

Fig 3 Velocity of nanoparticles generated by laser ablation at different pulse energies. (b) Velocity distributions of nanoparticles generated at $20 \mu \mathrm{J}$ and $160 \mu \mathrm{J}$ pulse energy.

Nanoparticles dispersed in liquids absorb and scatter the incident laser pulses. Depending on the irradiation parameters and particle cross section, a change in the morphology of the particles or deagglomeration can take place $[11,12]$. This leads to a progressive change of the colloids during the ablation process. This inter-pulse effect [2] was observed by measuring the particle size variation while increasing the number of pulses. The results are shown in Figure 4a. The same light scattering velocimetry method, based on the Brownian motion and Stokes-Einstein equation, was used to determine the hydrodynamic diameter of each particle detected. 


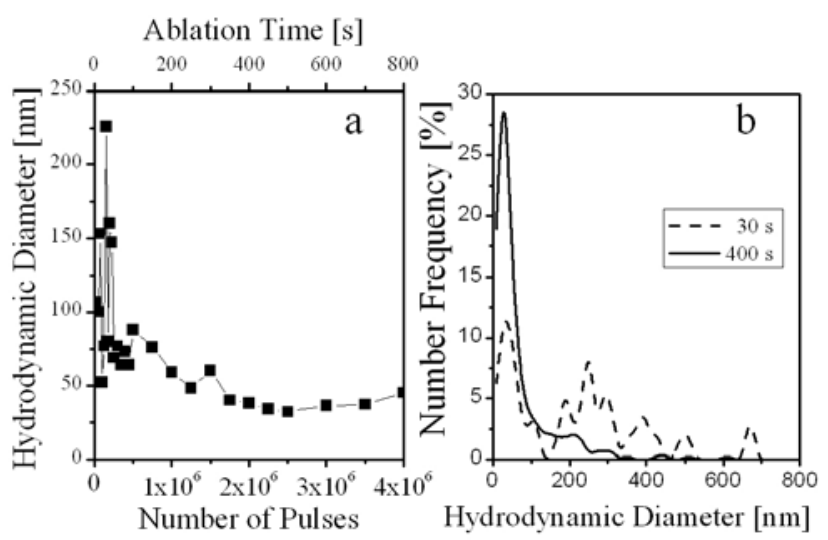

Fig 4 (a) Ag nanoparticle hydrodynamic diameter, represented by the percentile 50 of each size distribution generated at different number of pulses by pulsed laser ablation at $15 \mathrm{~mJ} / \mathrm{cm}^{2}$. (b) Nanoparticle size distributions generated after 30 s $\left(15 \times 10^{4}\right.$ pulses) and $400 \mathrm{~s}\left(2 \times 10^{6}\right.$ pulses $)$ ablation process.

At the beginning of the ablation, the particle size distributions is broad (Figure $4 \mathrm{~b}$ ) and the percentile-50 diameter $\left(\mathrm{d}_{50}\right)$ of each distribution varies strongly between 50 and $220 \mathrm{~nm}$ until $5 \cdot 10^{5}$ pulses are reached. For a higher number of pulses, the particle size stabilizes below $50 \mathrm{~nm}$, which is due to interaction of the freshly generated colloidal particles with the incident laser light. This is in accordance with UV-VIS spectra, showing a sharpening of the surface plasmon resonance band after irradiating the colloid with fslaser pulses, so that a deagglomeration effect can be assumed. In conclusion, studies on fs-laser generation of silver nanoparticles in liquids should always involve process times longer than 400 seconds (at a repetition rate of $5 \mathrm{kHz}$ ) in order to investigate stable process conditions.

Laser-generated nanoparticles show potential for medical applications since they are free of impurities and toxicities. Moreover, the electrical properties of such metal particles permit a directionally controlled diffusion under external electric fields and therefore deposition on conducting surfaces. The development of new techniques for guiding tissue growth is leading to implementation of nanostructures and nanomaterials. Surface roughness has been shown to be a decisive parameter in cell growth and adherence and tends to be enhanced by nanostructures rather than by microstructures [13]. For this reason, the deposition of nanoparticles on surfaces or scaffolds becomes a rapid method which can fit individual specifications in profile characteristics.

As metal nanoparticles generated by laser ablation are superficially negatively charged [14], they will migrate under the force of an external electric field if its magnitude is high enough to compensate the Stokes frictional force and Brownian motion. The coating of conducting surfaces by charged colloids is known as electrophoretic deposition and its application to nanoparticles is a powerful technique to generate nanotextures.

In order to control the coating thickness or number of particles per surface area, the electrophoretic deposition parameters have to be determined. Electric field, concentration and deposition time can be varied externally. The intrinsic parameter of the colloidal system is the response of the particle to the applied field, and needs to be experimentally determined, as it depends on material, particle size distribution and continuous medium. Implementing two gold electrodes in the chamber used for particle tracking analysis, the electrokinetic properties can be studied (Figure $2 b$ ).

The velocity distributions of gold nanoparticles dispersed in deionized water were measured for increasing electric field. The applied voltage was varied from 0 to $15 \mathrm{~V}$, while keeping electrode distance constant $(15 \mathrm{~mm})$ (Figure 5). The distributions are narrowed in the presence of external field, as the random walk of the particles is confined. At electric fields over $2 \mathrm{~V} / \mathrm{cm}$ the electrostatic and frictional forces are balanced and the nanoparticles begin to move along the field. In the range of $2-7 \mathrm{~V} / \mathrm{cm}$, the particle speed depends linearly with the electric field so mass flux can be easily controlled at a fixed concentration. Above a critical electric field magnitude $(7-10 \mathrm{~V} / \mathrm{cm})$, the electrophoretic mobility coefficient is not constant and convection or hydrolysis disturbs the migration phenomena of the particles within the water.

Platinum iridium nanoparticles were generated by fslaser ablation in acetone. The size distribution, measured by SEM over 100 particles, resulted to be lognormal with maximum at $26 \pm 10 \mathrm{~nm}$. Electrophoretic velocitiy measurement showed a similar behavior to the gold colloid. However, the threshold electric field for electrophoretic displacement is higher as an initial drift was present in the solution (Inset in Figure 5).

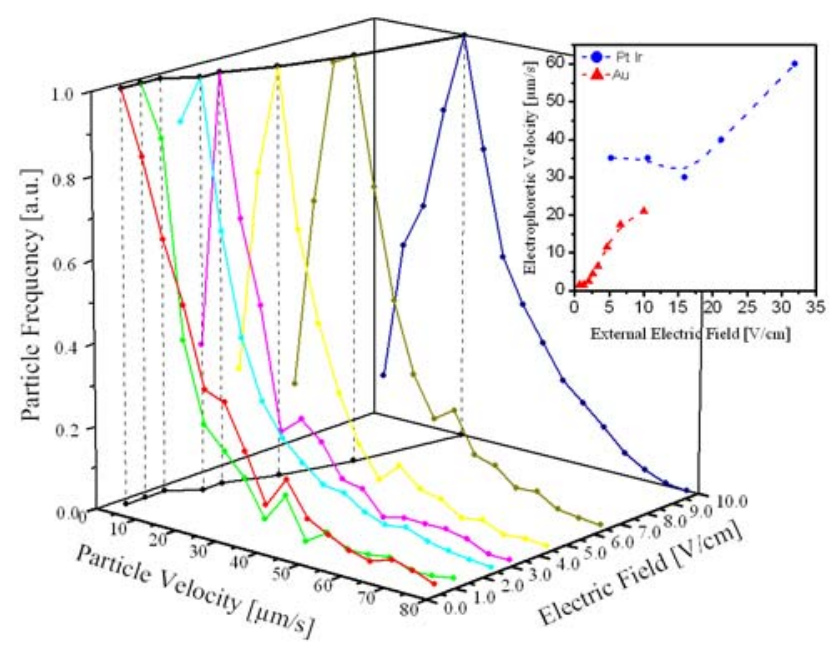

Fig 5 Electrophoretic velocity distributions of gold nanoparticles dispersed in deionized water for different electric field magnitudes. In the inset the most frequent velocity of gold nanoparticles in water (triangles) and platinum iridium nanoparticles in acetone (circles) plotted against the applied electric field.

Since the electrical field lines are normal to the surface of charge objects, complex 3-dimensional structures can be coated. As an example, microstructures coated with platiniridium and gold nanoparticles using electrodeposition of laser-generated metal nanoparticles are shown in Figure 6. 


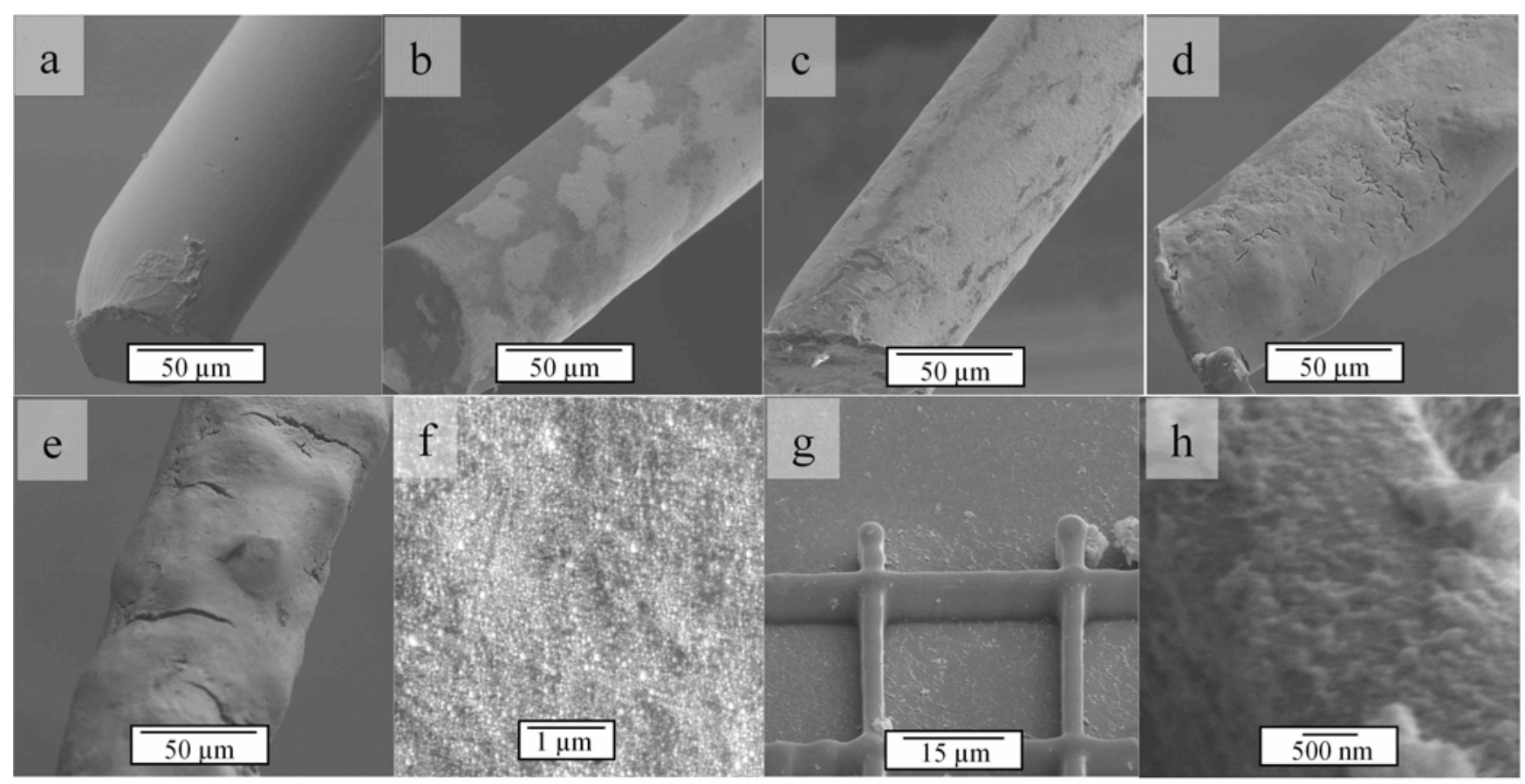

Fig 6 Scanning electron microscope images of microstructures coated with fs-laser generated (a-f) platinum iridium nanoparticles and (gh) gold nanoparticles using electrodeposition. The evolution of the coating texture is shown in pictures b, c, d and e for $20,30,40$ and 60 min deposition time respectively. Homogeneity of the coated layer with well-differentiated PtIr-particles is noticeable in f. Goldsputtered polymer microgrid is presented in $\mathrm{g}$, and the resulting surface appearance after deposition of gold nanoparticles in $\mathrm{h}$.

Surface of the microstructures before electrodeposition experiments can be seen in Figure 6a (platinum iridium neural electrode) and $6 \mathrm{~g}$ (gold sputtered polymer microgrid). Micrographs from figure $6 \mathrm{~b}-6 \mathrm{e}$ show how the coating texture varies with the treatment duration $(20,30,40$ and 60 min respectively). Figure $6 \mathrm{f}$ shows a close-up image of the deposited laser-generated platinum iridium nanoparticles. Micrograph in Figure $6 \mathrm{~h}$ shows electrodeposited gold nanoparticles on microgrid. For comparison, the original microstructure (without nanoparticles) is shown in Figure 6-g.

In summary, the dynamics of the nanoparticle generation process by laser ablation in water has been studied for a better understanding of the process. It has been found that the hydrodynamic size of the particles changes during the prolonged ablation and reaches a more stable level after 400 seconds. This indicates that post-irradiation of nanoparticles formed after a laser pulse leads to deagglomeration or fragmentation of the freshly produced colloid.

Since metal nanoparticles generated using the method of laser ablation in liquid are charged, their mobility characteristics in an electric field are of interest in order to study their stability and possible application using electrodeposition technique. We showed that the velocity of the nanoparticle motion directed in an electric field can be controlled by the field strength.

The monitoring technique and process chamber presented in this paper allow to generate the nanoparticles and study the process and material characteristics at the same time: the laser ablation in liquids, in-process study of the size of the generated particles and investigation of the particle motion in an electric field.

In order to outline possible future applications of this technique, we have demonstrated electrophoretic deposition to be a rapid and effective method to coat 3-dimensional sur- faces of electrodes for neural stimulation with laser generated nanoparticles.

\section{Author's Contributions}

Design, experiments and studies on particle tracking during ablation and on electrophoretic mobilities where carried out by AMM. JJ has proceeded with generation and electrophoretic coating of neural electrodes, previously selected and prepared by KS. Conception and supervision of improvement of medical implants by nanotexturing are due to JKK. SB has designed the study, supervised and promoted the collaboration of both institutions.

\section{Acknowledgments}

We thank Pere Maciá Rosell for the chambers design and construction. Sincerely thankful are we to the Deutsche Forschungsgemeinschaft for financial support of nanoparticle generation under the project CH197-8-1and Excellence Cluster REBIRTH for electrophoretic deposition.

\section{References}

[1] N. N. Nedialkov, S. E. Imamova, P. A. Atanasov, P. Berger and F. Dausinger. Appl. Surf. Sc. 247, (2005) 243.

[2] A. V. Kabashin and M. Meunier: J. Appl. Phys., 94, (2003) 7941.

[3] T. Tsuji, K. Iryo, N. Watanabe and M. Tsuji: Appl. Surf. Sc. 202, (2002) 80-85.

[4] C.H.Fan, J.Sun and J.P. Longtin: J. Appl. Phys, 91, (2002) 2530.

[5] C. Wang, Y. Fu, Z. Zhou, Y. Cheng and Z. Xu; Appl. Phys. Lett. 90, (2007) 181119.

[6] A. Couairon and A. Mysyrowicz: Phys. Rep. 441, (2007) 47. 
[7] S. Barcikowski, A. Menéndez-Manjón, B. Chichkov, M. Brikas, and G. Račiukaitis. Appl. Phys. Lett. 91, 083113 (2007), 083113.

[8] S. H. Ko, H. Pan, D.J. Hwang, J. Chung, S. Ryu, C. P. Grogoropuolos. J. App. Phys. 102, (2007) 093102.

[9] S. Amoruso, G. Ausanio, R. Bruzzese, L. Gragnaniello, L. Lanotte, M. Vitello, X. Wang. Appl. Surf. Sc. 252, (2006) 4863.

[10]J. König, S. Nolte,A. Tünnermann. Opt. Express 13, 26 (2005), 10597.

[11]S. Besner, A. Kabashin and M. Meunier. Appl. Phys. Lett. 89, (2006) 233122.

[12]A. Takami, H. Kurita and S. Koda: J. Phys. Chem B, 103, (1999) 1226.

[13]T. J. Webster, L. S. Schadler,R. W. Siegel, R. Bizios. Tiss. Eng. 7-3, (2001) 291.

[14]M. Shoji, K. Miyajima and F. Mafuné. J. Phys. Chem C 112, (2008) 1929.

(Received: June16, 2008, Accepted: May 7, 2009) 